\title{
[C ${ }^{\mathrm{Ar}} \mathrm{Ni}\{\mathrm{Ga}($ nacnac)$\}]$ : An open-shell nickel(I) complex supported by a gallium(I) carbenoid $\left(\mathrm{Cp}^{\mathrm{Ar}}=\mathrm{C}_{5}\left(\mathrm{C}_{6} \mathrm{H}_{4}-4-\right.\right.$ Et $)_{5}$, nacnac $\left.=\mathrm{HC}\left[\mathrm{C}(\mathrm{Me}) \mathrm{N}\left(\mathrm{C}_{6} \mathrm{H}_{3}-2,6-i \mathrm{Pr}_{2}\right)\right]_{2}\right)$
}

Uttam Chakraborty, ${ }^{a}$ Bernd Mühldorf,,${ }^{a}$ Niels van Velzen, ${ }^{b}$ Bas de Bruin, ${ }^{c}$ Sjoerd Harder,,${ }^{b, d}$ and Robert Wolf*a

\begin{abstract}
${ }^{a}$ University of Regensburg, Institute of Inorganic Chemistry, D-93040 Regensburg; E-mail: robert.wolf@ur.de; http://www.uni-regensburg.de/chemistry-pharmacy/inorganic-chemistrywolf/index.html; ${ }^{b}$ Stratingh Institute for Chemistry, Nijenborgh 4, 9747 AG Groningen, The Netherlands, ${ }^{c}$ University of Amsterdam, van 't Hoff Institute for Molecular Sciences, Science Park 904, 1098 XH Amsterdam, Netherlands, ${ }^{d}$ Friedrich Alexander University ErlangenNürnberg, Inorganic and Organometallic Chemistry, D-91058 Erlangen
\end{abstract}

\section{Supporting information contains}

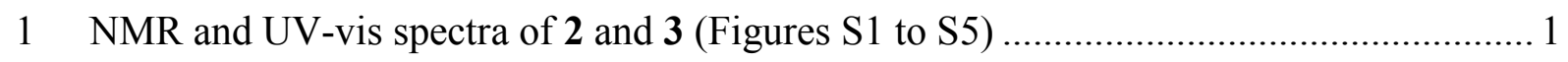

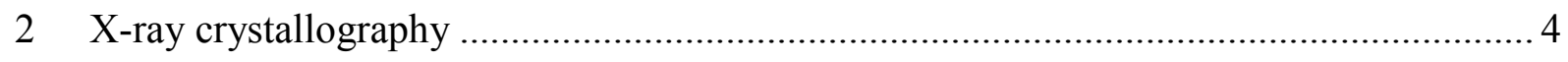

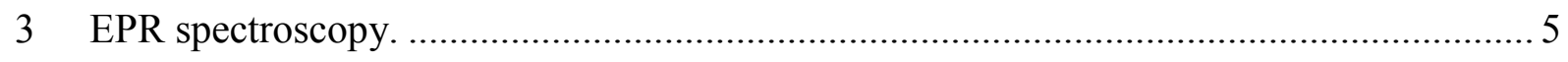

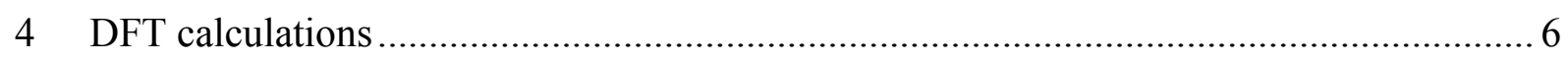

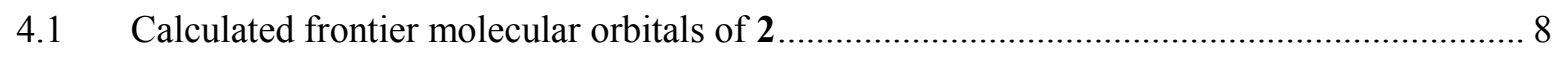

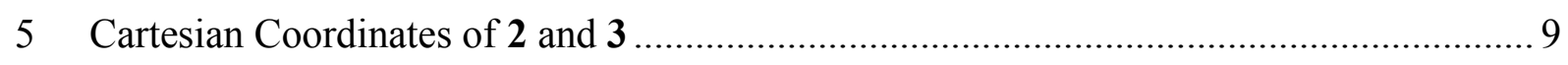

5.1 Cartesian Coordinates of $\mathbf{2}$ (optimized at the B3LYP / def2-TZVP level of theory) ............. 9

5.2 Cartesian Coordinates of $\mathbf{3}$ (optimized at the B3LYP / def2-TZVP level of theory) ............ 11 


\section{NMR and UV-vis spectra of 2 and 3 (Figures S1 to S5)}

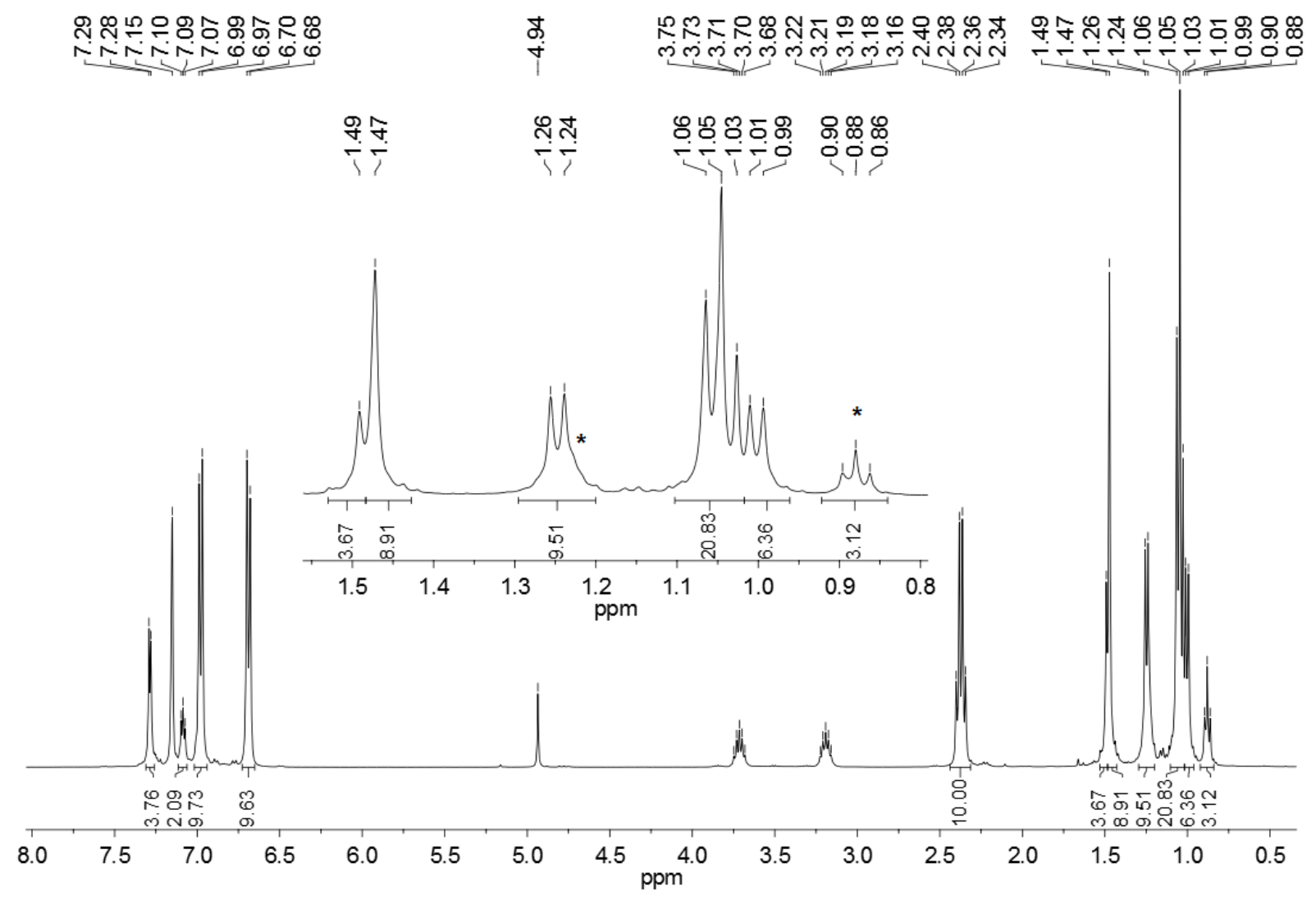

Figure S1. ${ }^{1} \mathrm{H}$ NMR spectrum of complex $2\left(400.13 \mathrm{MHz}, \mathrm{C}_{6} \mathrm{D}_{6}, 300 \mathrm{~K}\right)$; * denotes the signal corresponding to $n$-hexane. 


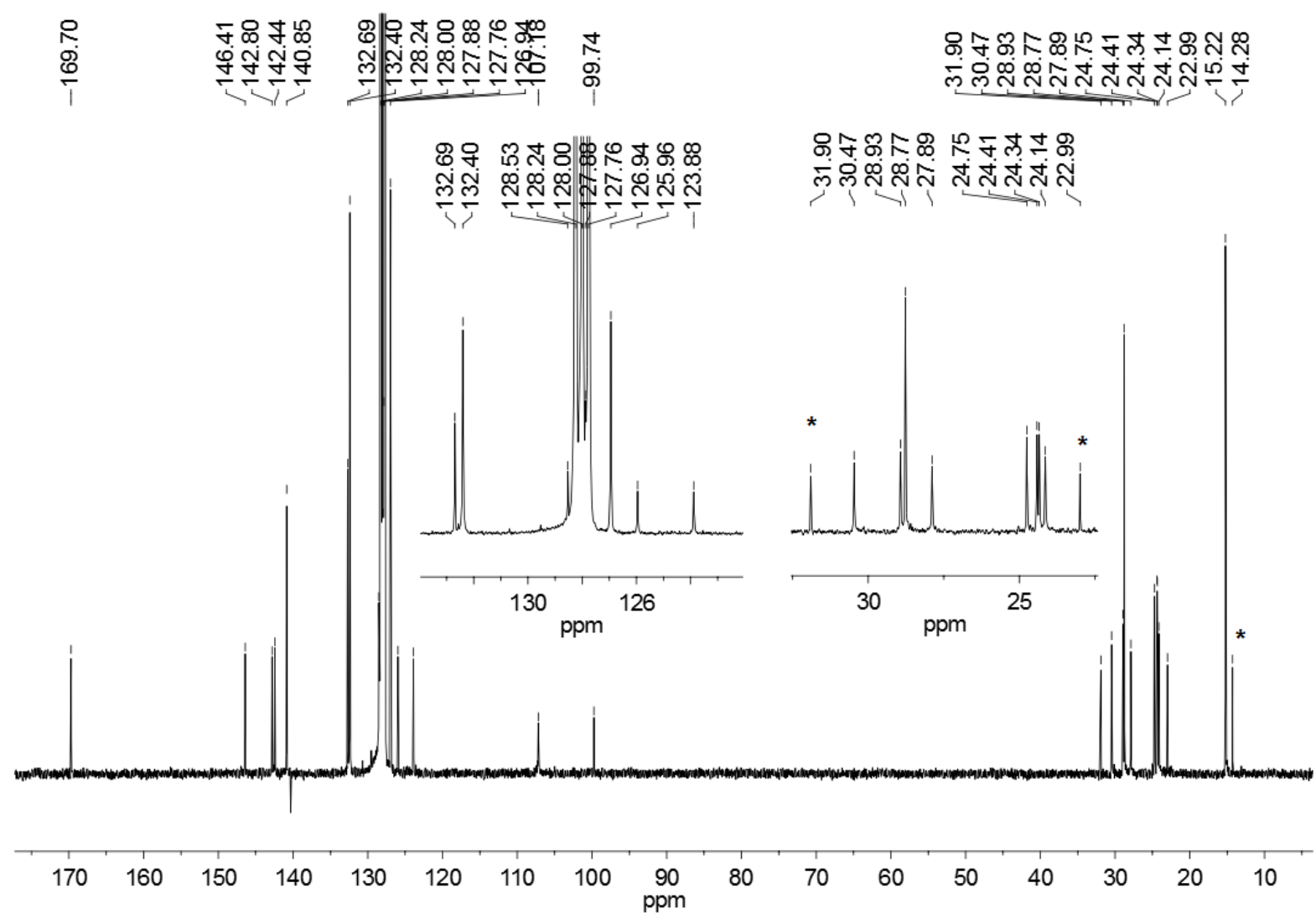

Figure S2. ${ }^{13} \mathrm{C}\left\{{ }^{1} \mathrm{H}\right\}$ NMR spectrum of complex $2\left(400.13 \mathrm{MHz}, \mathrm{C}_{6} \mathrm{D}_{6}, 300 \mathrm{~K}\right) ;{ }^{*}$ denotes the signal corresponding to $n$-hexane.

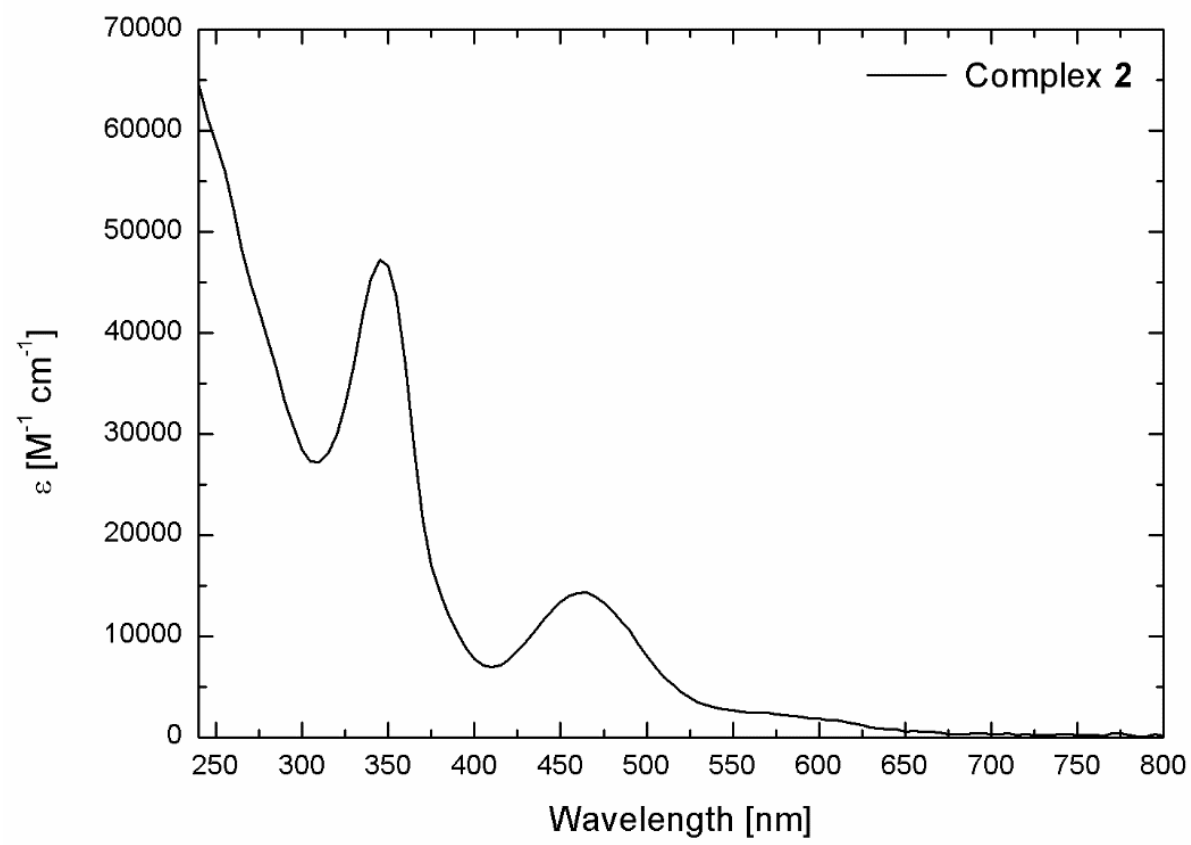

Figure S3. UV/vis spectrum of complex 2 in cyclohexane. 


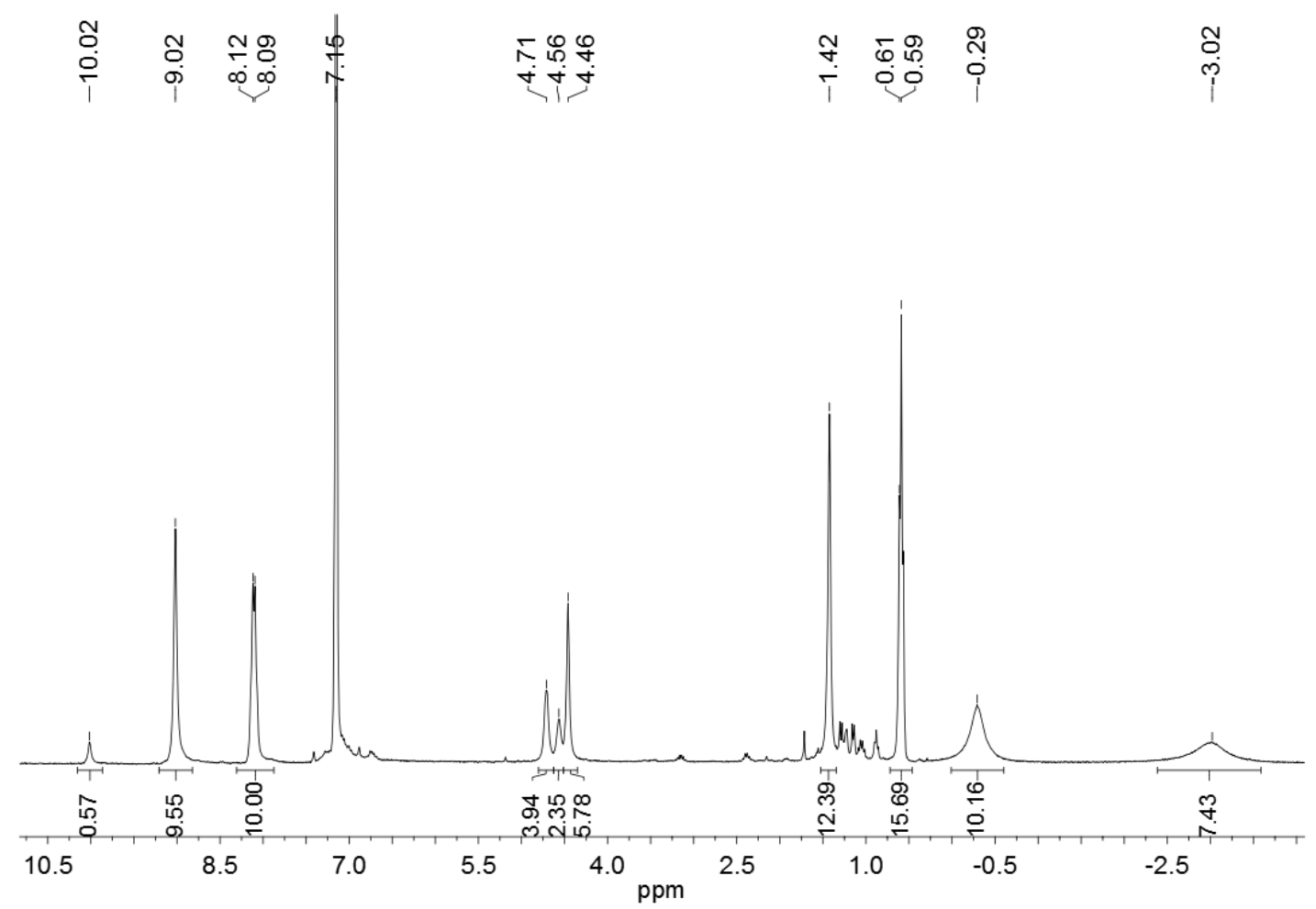

Figure S4. ${ }^{1} \mathrm{H}$ NMR spectrum of complex 3 (400.13 MHz, $\left.\mathrm{C}_{6} \mathrm{D}_{6}, 300 \mathrm{~K}\right)$.

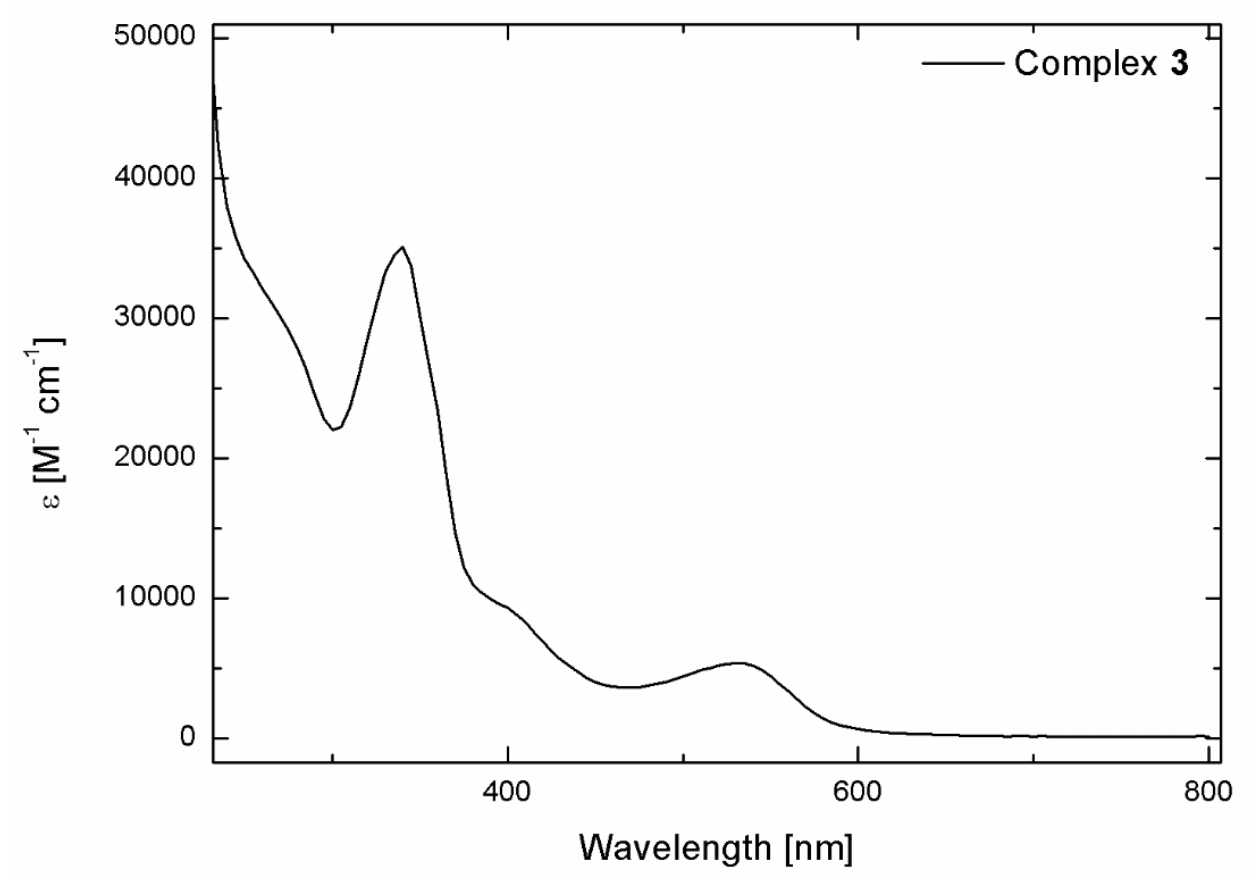

Figure S5. UV/vis spectrum of complex 3 in cyclohexane. 


\section{X-ray crystallography}

Dark red single crystals suitable for X-ray measurements were obtained by slow evaporation of the $n$-hexane solution of $\mathbf{2}$ and diffusion of $n$-hexane into a benzene solution of $\mathbf{3}$ at room temperature. The crystals were processed at an Agilent Technologies SuperNova single source at offset, atlas diffractometer with microfocus $\mathrm{Cu}$ radiation (2) and an Agilent Technologies Xcalibur, AtlasS2, Gemini ultra device employing microfocus $\mathrm{Cu}$ radiation (3). The CrysAlis software was used to apply gaussian (2) or analytical (3) absorption corrections. ${ }^{1}$ Using Olex2, the structures were solved with direct methods by ShelXT and refined with ShelXL using least squares minimization. ${ }^{3}$ PLATON/SQUEEZE was used for the refinement of $2 .{ }^{4}$ The asymmetric unit of complex 2 contains three crystallographically independent molecules. Important crystallographic data for these three molecules are given in Table S1. Details of the structure determinations are given in Table S2 of the supporting information. The crystallographic information files (CIF) have been deposited at the CCDC, 12 Union Road, Cambridge, CB21EZ, U.K., and can be obtained on request free of charge, by quoting the publication citation and deposition numbers CCDC 1441729 and 1441730.

Table S1. Crystallographic data for the three different molecules of 2 in the asymmetric unit

\begin{tabular}{|c|c|c|c|c|}
\hline & Molecule 1 & Molecule 2 & Molecule 3 & Average $^{a}$ \\
\hline $\mathrm{Cp}^{\mathrm{Ar}}$ (centroid)-Ni & $1.767(1)$ & $1.783(2)$ & $1.774(2)$ & $1.775(5)$ \\
\hline $\mathrm{Ni}-\mathrm{Ga}$ & $2.2569(6)$ & $2.2840(6)$ & $2.2655(8)$ & $2.2688(8)$ \\
\hline $\mathrm{Ni}-\mathrm{Br}$ & $2.3444(5)$ & $2.3652(6)$ & $2.3636(5)$ & $2.3577(7)$ \\
\hline $\mathrm{Ga}-\mathrm{Br}$ & $2.6905(5)$ & $2.6679(6)$ & $2.6999(6)$ & $2.6861(9)$ \\
\hline $\mathrm{Cp}^{\mathrm{Ar}}$ (centroid)-Ni-Ga & $149.917(2)$ & $153.461(2)$ & $150.820(2)$ & $151.40(1)$ \\
\hline $\mathrm{Ni}-\mathrm{Br}-\mathrm{Ga}$ & $52.72(2)$ & $53.57(2)$ & $52.65(2)$ & $52.98(3)$ \\
\hline $\mathrm{N}-\mathrm{Ga}-\mathrm{N}$ & $94.9(1)$ & $93.9(1)$ & $94.0(1)$ & $94.3(3)$ \\
\hline
\end{tabular}

${ }^{a}$ The unweighted mean value $\mathrm{x}_{\mathrm{u}}$ of bond lengths and angles are given, the standard deviation $\sigma$ of $x_{u}$ was calculated using the equation $\sigma^{2}=\Sigma\left(x_{i}-x_{u}\right)^{2} /\left(n^{2}-n\right)$, where $x_{i}$ is the individual value and $\mathrm{n}=$ number of elements. 
Table S2. Crystallographic data of 2 and 3

\begin{tabular}{|c|c|c|}
\hline & 2 & 3 \\
\hline Empirical formula & $3\left(\mathrm{C}_{74} \mathrm{H}_{86} \mathrm{BrGaN}_{2} \mathrm{Ni}\right)$ & $\mathrm{C}_{74} \mathrm{H}_{86} \mathrm{GaN}_{2} \mathrm{Ni}$ \\
\hline Formula weight & 3635.35 & 1131.87 \\
\hline Temperature/K & $123(1)$ & $124(1)$ \\
\hline Crystal system & monoclinic & monoclinic \\
\hline Space group & $P 2_{1} / \mathrm{n}$ & $P 2 / \mathrm{c}$ \\
\hline $\mathrm{a} / \AA$ & $23.8919(4)$ & $24.54660(18)$ \\
\hline $\mathrm{b} / \AA \AA$ & $22.8702(4)$ & $11.25767(8)$ \\
\hline $\mathrm{c} / \AA \AA$ & $38.3227(7)$ & $22.79171(15)$ \\
\hline$\alpha /^{\circ}$ & 90 & 90 \\
\hline$\beta /{ }^{\circ}$ & $97.7389(19)$ & $98.9460(7)$ \\
\hline$\gamma /{ }^{\circ}$ & 90 & 90 \\
\hline Volume $/ \AA^{3}$ & $20749.3(7)$ & $6221.59(8)$ \\
\hline Z & 4 & 4 \\
\hline$\rho_{\text {calc }} \mathrm{mg} / \mathrm{mm}^{3}$ & 1.164 & 1.208 \\
\hline$\mu / \mathrm{mm}^{-1}$ & 1.782 & 1.222 \\
\hline $\mathrm{F}(000)$ & 7656 & 2412.0 \\
\hline Crystal size $/ \mathrm{mm}^{3}$ & $0.274 \times 0.14 \times 0.076$ & $0.381 \times 0.153 \times 0.067$ \\
\hline $2 \Theta$ range for data & 3.386 to $74.021^{\circ}$ & 7.292 to $132.042^{\circ}$ \\
\hline Index ranges & $\begin{array}{l}-29 \leq \mathrm{h} \leq 21 \\
-28 \leq \mathrm{k} \leq 28 \\
-46 \leq 1 \leq 47\end{array}$ & $\begin{array}{l}-29 \leq \mathrm{h} \leq 28 \\
-12 \leq \mathrm{k} \leq 8 \\
-26 \leq 1 \leq 27\end{array}$ \\
\hline Reflections collected & 173427 & 43690 \\
\hline Independent reflections & $41200\left(R_{\mathrm{int}}=0.0328\right)$ & $10697\left(R_{\mathrm{int}}=0.0260\right)$ \\
\hline Data/restraints/parameters & $41200 / 0 / 2179$ & $10697 / 0 / 728$ \\
\hline Goodness-of-fit on $\mathrm{F}^{2}$ & 0.966 & 1.029 \\
\hline $\mathrm{R}_{1}, \mathrm{wR}_{2}[\mathrm{I}>=2 \sigma(\mathrm{I})]$ & $0.0509,0.1450$ & $0.0330,0.0818$ \\
\hline $\mathrm{R}_{1}, \mathrm{wR}_{2}$ [all data] & $0.0643,0.1570$ & $0.0382,0.0860$ \\
\hline $\begin{array}{l}\text { Largest diff. peak/ } \\
\text { hole / e } \AA^{-3}\end{array}$ & $1.358 /-0.700$ & $0.947 /-0.457$ \\
\hline $\mathrm{CCDC}$ & 1441729 & 1441730 \\
\hline
\end{tabular}

\section{EPR spectroscopy.}

The experimental X-band EPR spectrum of 3 was recorded on a Bruker EMX spectrometer (Bruker BioSpin Rheinstetten) equipped with a He temperature control cryostat system (Oxford Instruments). Simulations of the EPR spectra were performed by iteration of the anisotropic $\mathrm{g}$ values and line widths using the EPR simulation program W95EPR developed by Prof. Dr. Frank Neese. The spectrum is shown in Figure S6. 


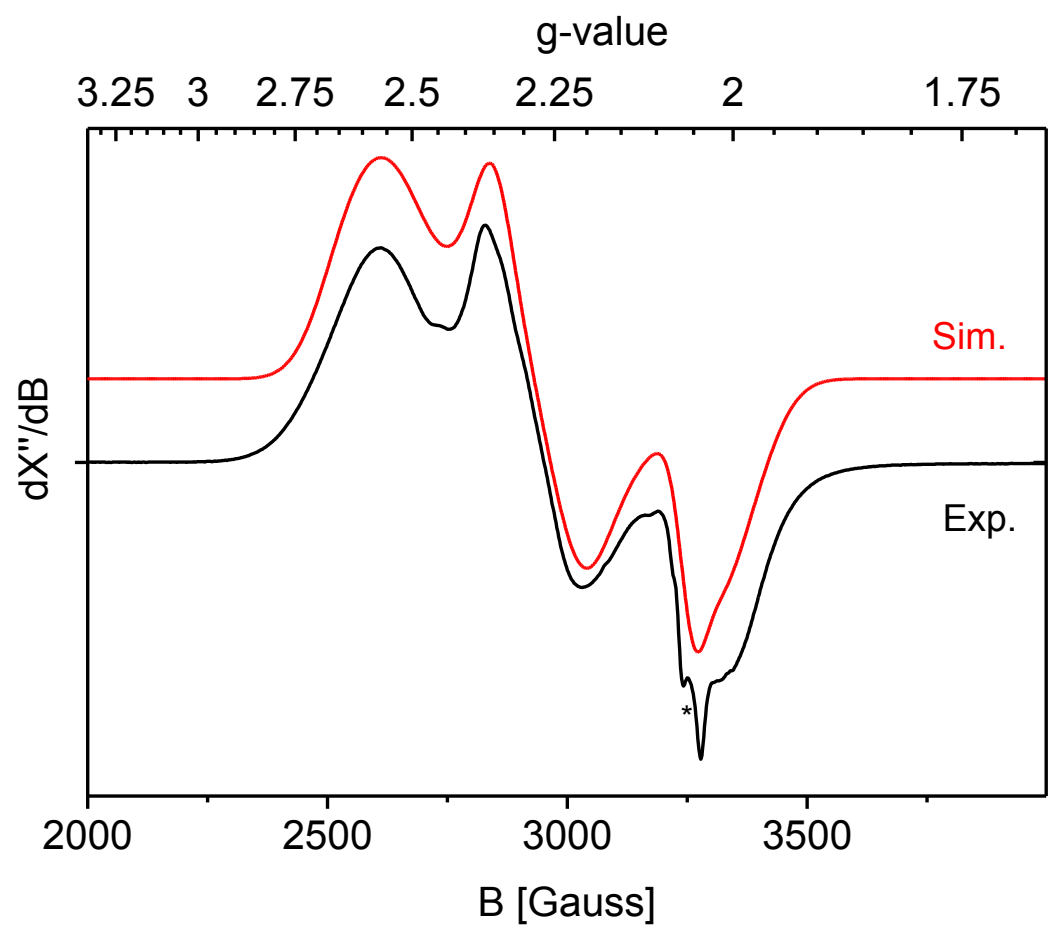

Figure S6. Experimental and simulated X-band EPR spectrum of $\mathbf{3}$ in frozen toluene recorded at $20 \mathrm{~K}$. Frequency $=9.363281 \mathrm{GHz}$, microwave power $=0.0633 \mathrm{~mW}, \mathrm{~T}=20 \mathrm{~K}$, modulation amplitude $=4$ Gauss. The sharp signal indicated with * appears to be a minor impurity. The simulated spectrum was obtained using the parameters shown in Table S3.

Table S3. Experimental and DFT calculated isotropic EPR parameters of 3.

\begin{tabular}{|l|c|c|c|c|c|c|}
\hline & $\mathbf{g}_{\boldsymbol{x}}$ & $\mathbf{g}_{\boldsymbol{y}}$ & $\mathbf{g}_{\boldsymbol{z}}$ & $\boldsymbol{A}_{\boldsymbol{x}}^{\mathbf{G a}}$ & $\boldsymbol{A}^{\mathbf{G a}}{ }_{\boldsymbol{y}}$ & $\boldsymbol{A}^{\mathbf{G a}}{ }_{z}$ \\
\hline Exp. $^{(\mathrm{a})}$ & 2.01 & 2.28 & 2.58 & $\mathrm{NR}^{(\mathrm{c})}$ & $\sim 130^{(\mathrm{d})}$ & $\mathrm{NR}^{(\mathrm{c})}$ \\
\hline$D F T^{(b)}$ & 2.117 & 2.291 & 2.403 & -64 & -92 & -75 \\
\hline
\end{tabular}

(a) Values from spectral simulation. Hyperfine interactions in MHz. Signs of the HFIs follow the DFT computations.

(b) DFT, Orca, b3-lyp, def2-TZVP. Hyperfine interactions in MHz.

(c) Not resolved

(d) Not resolved, estimated from simulation of the line shape.

\section{DFT calculations}

The calculations on $\mathbf{2}$ and $\mathbf{3}$ were performed using the ORCA program package (version 3.0.2.). ${ }^{5}$ The B3LYP density functional and the Ahlrichs def2-TZVP basis set were employed for all atoms. $^{6,7}$ The RI approximation was used. The Ahlrichs Coulomb fitting basis for the TZVP basis for all atoms $(\mathrm{TZV} / \mathrm{J})$ and the atom-pair-wise dispersion correction to the DFT energy with Becke-Johnson damping ( $\mathrm{d} 3 \mathrm{bj})$ were applied. ${ }^{8}$ The nature of the stationary points was verified by numerical frequency analyses. Reduced orbital charges and spin densities were calculated according to the Löwdin population analysis. ${ }^{9}$ Molecular orbitals and the spin density 
plots were visualized via the program Gabedit. ${ }^{10}$ The isosurface value is set to 0.05 for all figures, except for the plot of the spin density of $\mathbf{3}$ (isosurface value 0.005 ).

EPR property calculations were performed with ORCA using the B3LYP functional and the def2-TZVP basis set (without dispersion corrections) using the ORCA optimized geometry of complex 3 .

Table S4. Experimental and calculated structural data for complexes 2 and 3.

\begin{tabular}{c|cc|ccc}
\hline & \multicolumn{2}{|c|}{ Bond length $(\AA)^{*}$} & \multicolumn{3}{c}{${\text { Bond angle }\left({ }^{\circ}\right)}$} \\
\cline { 2 - 6 } & Ni-Cp ${ }^{\mathrm{Ar}}$ (centroid) & $\mathrm{Ni}-\mathrm{Ga}$ & $\mathrm{Cp}^{\mathrm{Ar}}($ centroid)-Ni-Ga & $\mathrm{Ni}-\mathrm{Ga}-\mathrm{N} 1$ & $\mathrm{Ni}-\mathrm{Ga}-\mathrm{N} 2$ \\
\hline $\mathbf{2}$ exp. & 1.775 & 2.2688 & 151.40 & 132.37 & 130.51 \\
$\mathbf{2}$ cal. & 1.806 & 2.258 & 152.00 & 132.17 & 130.46 \\
$\mathbf{3}$ exp. & 1.792 & 2.291 & 164.62 & 122.12 & 145.75 \\
$\mathbf{3}$ cal. & 1.858 & 2.292 & 156.89 & 121.68 & 145.23 \\
\hline
\end{tabular}




\subsection{Calculated frontier molecular orbitals of 2}
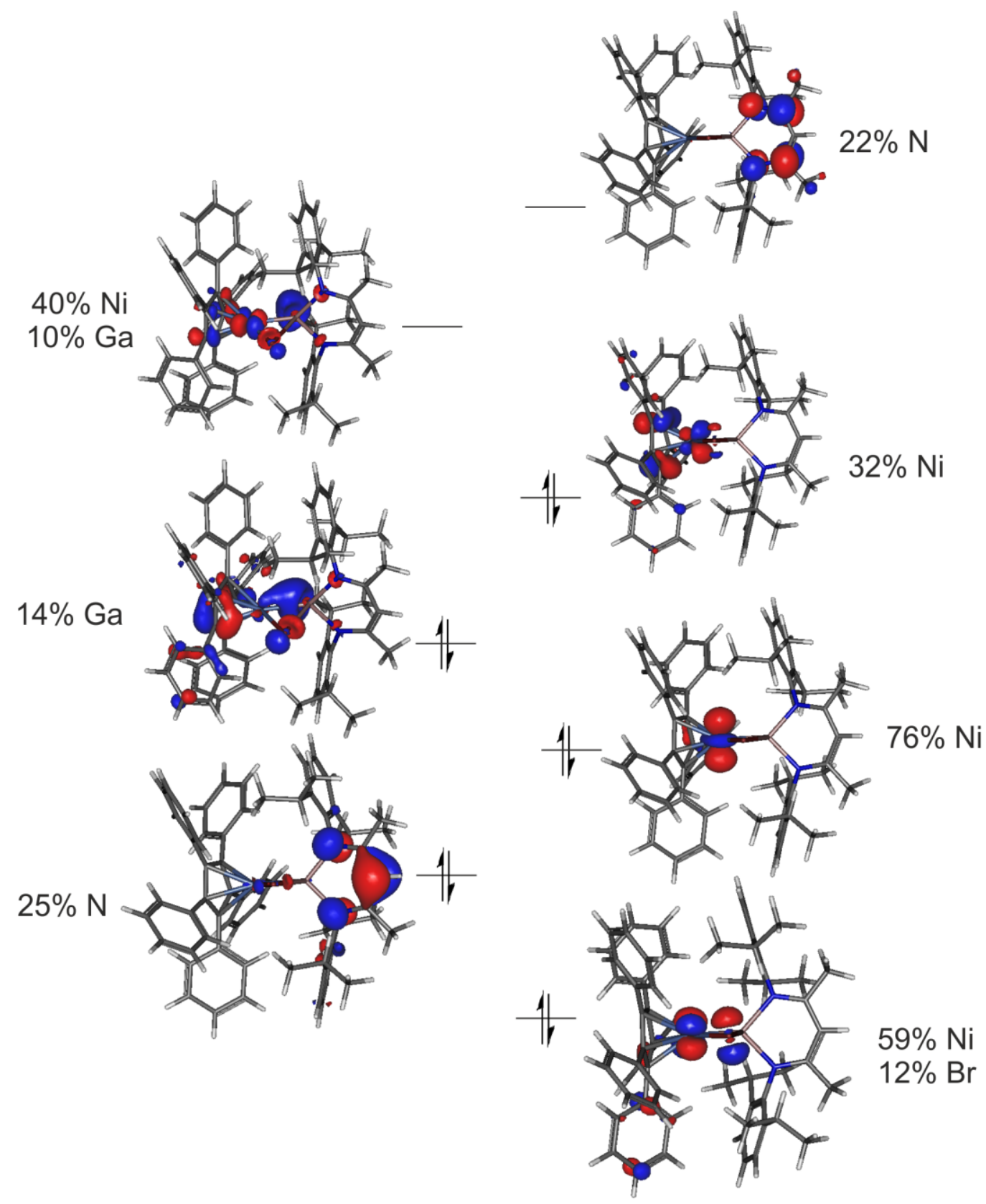

Figure S7. Calculated (B3LYP) frontier Kohn-Sham orbitals of 2 with reduced Löwdin atomic orbital populations per molecular orbital. 


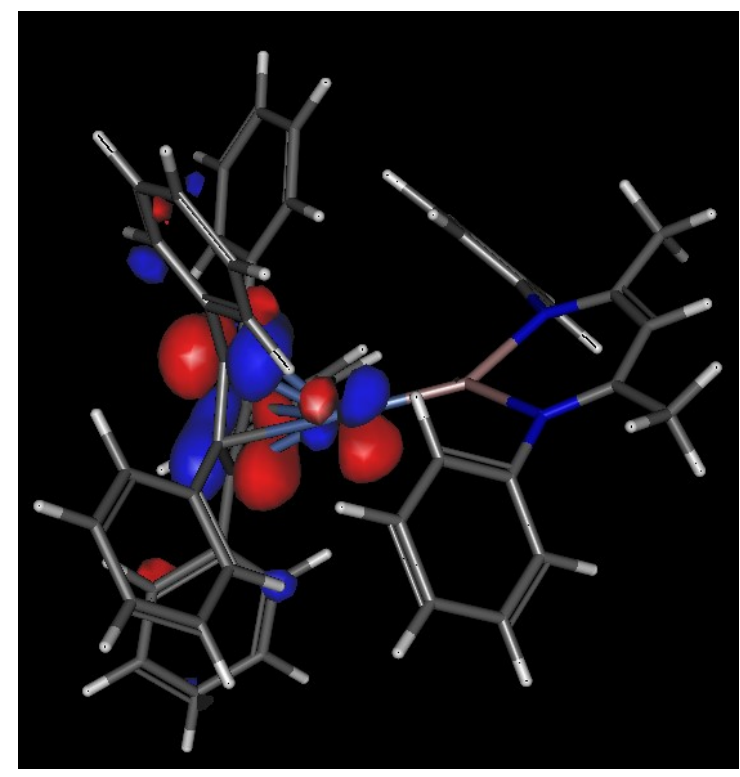

Figure S8. The SOMO spin up (no 213, $-3.86 \mathrm{eV}$ ) of $\mathbf{3}$ calculated at the B3LYP / def2-TZVP level of theory; a Löwdin population analysis indicates that this orbital has $20 \%$ nickel character. ${ }^{9}$

\section{Cartesian Coordinates of 2 and 3}

\subsection{Cartesian Coordinates of 2 (optimized at the B3LYP / def2-TZVP level of theory)}

Energy $=-8594.954781557273$ Hartree

$\mathrm{Br} 30.43803820622040$ Ga 30.44949160719305 Ni 32.07943194825220

N 28.63892544720012

N 30.09729340597880

C 33.82436687850910

C 33.19823140947953

C 33.77265716445449

C 34.26033619240809

C 27.59805676031249

C 28.43985943671878

C 28.87247291879994

C 31.22234396502529

C 34.26001761432864

C 32.88493901108250

C 33.14750666352168

C 33.96210794110166

C 35.12709546990437

C 27.71296279488878

C 26.20162643440556

C 28.02992857621915

C 28.64484763657754

C 28.63773289404066

C 31.50836105433062

C 32.00753487882140

C 35.56616437490566

C 33.44543090728241

C 31.58816731841190

C 33.95489656108685

C 32.55784402671539

C 34.39494245581839

C 33.72860540182202

C 36.28450958002863

C 34.86426176895866
21.66175742679805 19.38098524746589 20.94069892469569 19.30062037411433 17.95844122040115 20.44404996641387 21.53189173774773 22.29045092903060 20.96580040484081

18.76824895167618 19.76965133937378 17.52887274559220 17.37558637528735 19.19344575047432 21.47822456576149 22.65744383065571 23.17975427643671 20.30543982009087 18.00319857283743 18.97580914478212 21.08530203832472 18.84622588794997 16.44218606365979 17.72846322690781 16.43425679192153 18.72824433575118 18.52062637946328 21.49334618877221 21.42813956831450 23.96397671374888 24.49742693804929 22.72079805102302 20.96362496449363 19.02755754798891
$-3.22403028056137$ $-1.78677408573464$ $-1.69003576901736$ $-0.99224557202801$ $-3.14089136312043$ $-0.64814914629189$ 0.09385086906185 $-2.00577184029197$

$-1.91333599676292$ $-1.63671956589180$ 0.35336129397276 $-3.41755874770943$ $-3.82003004616192$ 0.00407545166790

1.53731711373368

$-0.74023164368167$

$-3.16262462805510$ $-2.90242236673087$ $-2.79290814553055$ $-1.10686059930676$ 0.61492890912515 1.39629129466329 $-4.43703930881965$ $-5.14962549685936$ $-3.12797667360012$ $-0.17846790620051$ 0.92201920432963 2.04260015292485 2.43613980885294 $-0.40627606139665$ $-2.99130485149772$ $-4.46148818452358$ $-3.33356785220179$ $-3.40057174937136$ 
C 27.88725548993038

C 27.77071004540623

C 28.36301257231382

C 29.11244475399106

C 30.72174692775155

C 32.56137981263531

C 31.70601846694825

C 33.04493085424239

C 36.04142025916655

C 33.92828939778023

C 31.36609004876528

C 33.73596756780458

C 31.76496920037326

C 32.77704942823424

C 34.55731235375147

C 33.88952243112745

C 37.15498749692541

C 35.74729684790152

C 28.90090611469416

C 26.46843181047865

C 27.91308305338049

C 30.11937006183172

C 27.92804191682528

C 31.61051053996048

C 30.10086050764173

C 33.31225845413544

C 32.91479844133918

C 30.52469163492495

C 35.23009500883445

C 32.43661972229960

C 31.20066057978727

C 32.21316169710040

C 34.29791179339085

C 36.89470450515743

C 31.41445760513756

H 26.78766850590022

H 25.51017909825035

H 25.87386715044589

H 26.15153361088401

H 28.14815166768716

H 27.96380472407413

H 29.55207517532457

H 36.22069627173823

H 32.43053761922290

H 30.75101869487980

H 34.96554098118131

H 34.59731096028899

H 33.40756751712051

H 36.49830174316538

H 33.96384897115202

H 28.12293537615530

H 27.44871659347254

H 28.49912388867141

H 29.61628854060348

H 29.91804765838550

H 32.79703083111093

H 31.41895499316581

H 33.65496562392321

H 37.05369595453376

H 33.28212317087704

H 30.35108067433988

H 34.57816362829275

H 31.56695349539181

H 33.39551214801271

H 34.88855792275903

H 33.68626519278375

H 38.03956509033112

H 35.53066119416047

H 28.84750902047407

H 29.91783090445081

H 28.71398579667243
22.13183061719578

21.44012965210016 19.24501222886181 17.42872706272174 18.77430121907471 17.07467708595242 16.05738031250599 15.80856960435275 17.63276069666372 17.44463534199439 21.47642441840194 21.41020252829173 24.65997502456491 24.54373749214015 25.33694344408183 23.55719276377643 20.36575024646791 18.42150104145667 23.25939916254441 22.70620722608971 20.52863840674944 16.91815798484645 16.45962918604574 19.97500122274630 18.20124639098911 16. 11322331054471 15.49184850808397 15.08014287440495 16.99326610446269 21.43863708216058 25.88328516436564 25.76948864615375 24.87404254059518 19.08380977103825 26.44282154543585 17.61391704157927 18.27159229984888 19.98553711538531 18.88115295269260 16.87060890036333 15.69576642236541 15.95509381395551 19.23639214172295 18.85884949483427 21.50965620870315 21.40465904357837 24.86833527311854 21.70197105195703 21.95304827786596 18.51197331302890 21.66132743218399 22.44911863099227 18.54664718685017 17.43504390263355 19.14117878924686 17.32903689408916 16.97350236093581 15.07602957636488 17.28764635239895 16.94977020638179 21.48663052808367 21.37311002678721 24.22392861000998 24.03271215729825 26.35640094563635 23.17850811780493 20.89771297337218 17.42974480595097 23.97873453702519 22.87690651217764 23.79289548470820
$-0.47293661313104$ 1.93955498491060 2.69996116986656 1.11369862721827 $-5.92028163718373$ $-5.78988709873529$ $-1.68661788677829$ $-3.81477160579720$ 0.53182640916754 1.65006818836788 3.41499857009438 3.80559814548243 $-1.32505926740983$ 0.84832762357229 $-4.08528272000869$ $-5.55436478583006$ $-4.23355323027951$ $-4.28445966936919$ $-0.25900360563192$ $-0.56644899885870$ 2.97127476269161 2.14795020185052 0.99350096358095 $-6.26013105283476$ $-7.20327292381987$ $-5.14008893702394$ $-0.94357715972750$ $-1.60006760456264$ 1.46027229302021 4.30029944626274 $-0.99708170082262$ 1.17474117920811 $-5.37076824940713$ $-4.70500162742258$ 0.25797657680663 $-3.18919675184220$ $-1.56278641246650$ $-1.35738563089137$ $-0.02448340032624$ $-5.31310448304075$ $-4.01743756613441$ $-4.75664810657455$ $-0.87073689331678$ 1.07442172112273 1.36021857928592 2.04966889387025 $-1.99633537253210$ $-4.60767412371094$ $-2.95249724012420$ $-3.09699517182205$ $-1.42582674309931$ 2.16168611153316 3.51443782238553 0.14787154622853 $-5.28275839817820$ $-6.81463339486110$ $-1.16055320377592$ $-3.30804688294184$ 0.36143743330446 2.36343086613090 3.78621965538143 4.48508608460837 $-2.29272115818928$ 1.57118897310148 $-3.93215435310228$ $-6.54822988263711$ $-4.56098088969489$ $-4.65318179118504$ $-1.07517722842190$ $-0.22908167674781$ 0.67513104791234 
H 26.18644693700012

H 25.72227246460876

H 26.41264433513735

H 27.68919175680122

H 29.63628793280001

H 30.89838818735833

H 30.59258002973013

H 27.35666769769792

H 28.28607787170795

H 27.25171069638199

H 32.05219206387458

H 31.02146326207360

H 32.41381764666286

H 30.87407468743634

H 29.48186245662967

H 29.47953104922179

H 34.11701537489996

H 33.20105984000659

H 32.67148850111855

H 33.77470178129760

H 30.73638354139616

H 29.59885579094083

H 30.35758866074701

H 35.60426288516783

H 32.26018433145817

H 30.57976445477000

H 32.40422825304788

H 34.41812708481193

H 37.57699261164778

H 30.96715131691047
23.22126764972523

21.93365077705379 23.43215939204168 20.82343295376385 16.68117478200057 17.65480525805116 16.00415185484086 16.44043129667300 15.44850287058833 16.74297591790907 20.38693181328713 20.76294591387240 19.69032303364760 17.89947715221060 18.95739972592047 17.32826451069151 15.60964917796860 14.50998787971882 15.37336043892930 16.15612111838876 14.18370789884895 15.51764881839519 14.77521972006184 16.14871511070575 21.42564496864560 26.39426180715223 26.19907156597904 25.53236709964784 18.60660334269845 27.39420455994436
0.35424188658289 $-0.75403011800301$ $-1.37995266170208$ 3.98897253122283 3.09789883843181 2.34297918435134 1.78447406492889 1.92432526926994 0.78888764702104 0.18836222587460 $-5.35773994966004$ $-6.73309875620401$ $-6.94286878077485$ $-7.91235550775901$ $-7.69040356106518$ $-7.00730459889417$ $-5.66044695595703$ $-1.32662072040984$ 0.11147897564772 $-1.01199635039345$ $-2.18724236389199$ $-1.96844118332497$ $-0.56523649633048$ 2.02486351838564 5.36862392059723 $-1.72247442871955$ 2.15038496616132 $-6.22178971880402$ $-5.39690809579663$ 0.51767625551587

\subsection{Cartesian Coordinates of 3 (optimized at the B3LYP / def2-TZVP level of theory)}

Energy = -5549.161663916568 Hartree

Ga 3.97665124933655 Ni 4.82264487315859 N 4.88717081045522

N 2.36965474553574

C 6.21535514444559

C 3.56286565775677

C 5.69696573771959

C 4.30901577049831

C 5.81138148198564

C 4.35372335215968

C 2.13031103149611

C 1.41468843978053

C 7.33040906233932

C 6.39082506795900

C 4.49411595144411

C 2.09883353069742

C 6.80572058763339

C 3.75624275853639

C 7.08122732039824

C 3.05142532707398

C 0.78068932866891

C 0.74362735479966

C 1.21912045086232

C 8.60662085685948

C 7.66621419230126

C 4.17985001018494

C 1.40701884598171

C 1.35654821873968

C 6.93832924219068

C 7.74198860996479

C 4.16853530536607

C 2.80955498333442

C 7.22308503588431
6.71648917272495 4.60887076028554 8.34149142810510 7.77362400475223 8.05176642236911 2.78058747407045 2.65461327941363 2.60898440901972 2.88433805500411 9.54740652828209 9.04463558007676 7.03189436859551 8.57644749545860 7.12663840673786 2.93597229475172 2.71431780755855 2.36726820611217 2.35793980202717 2.88494612780547 9.85877642130151 9.64439153426541 5.96758684982338 7.30173270511387 8.19468092345917 6.73497020895630 3.06318436602501

3.57320892689523

1.75359185587097

3.03876283519093

1.37705874562862

1.23749414783705

3.21075710137191

2.12112752719016
11.41974817861982

11.72984783939748

12.17598342372006

10.91703758441430

12.60726942236804

12.11507081846902

11.21951533280057

10.90039955530885

12.63232416286571

12.32453049040856

11.24900555326772

10.15283769494025

11.95626224175604

13.63579139093606

13.18831499907420

12.25313276032268

10.29245234003109

9.55713246109574

13.37791128378098

11.91100540715637 10.93596980944815 10.74652869779369 8.79936924596560 12.35291560223148 14.01814085186309 14.62130272285525 13.11559222334940 11.55580823249311 9.07379917679313 10.60863536649822

8.82983122954503

8.98461254553408

14.54206385243286 
C 8.19849352455443

H 2.71524043227583

H 0.66077618900811

H 0.65433571196509

H -0.01565000238400

C -0.11248932368766

C 0.34585872063406

C 8.77785264705683

H 9.46900958832764

H 7.79076565403615

C 3.31294963429251

C 4.76568906187553

H 1.95982972077841

C 0.03506157155708

C -0.01841534624954

H 6.22639266708018

C 7.96064598411227

H 7.65870370843379

C 8.76964739041796

H 4.90316552442951

C 3.65344531185505

H 2.49013788363455

C 2.28623080443303

H 6.37279878842658

C 8.44069960289815

C 9.41947325739311

H 8.10728680842497

H -0.59971897644703

C -0.31799108744422

H 0.18955992164220

H 9.77083338294693

H 2.85772607804228

C 3.02729688521466

H 5.45321476167944

C 4.48475544403290

H -0.47382291985758

C -0.68815620385959

H 8.04409664600690

C 8.88280491355975

H 9.48289270702922

C 2.70430818860274

H 3.99395843390773

H 1.55559122483354

H 8.52710988511350

C 9.55016809999147

H 10.27260794999868

H -0.97938859554770

C 3.60670203178835

H 2.35081718441863

H 4.94978265133858

C 5.14520649054870

H 5.82008667962347

H 5.75933790513914

H 4.47788094348859

H 7.19421541997816

H 5.51820038274620

H 0.90841749153507

H 1.76594919946908

H 9.67966764560537

H 2.29790711293478

H 10.50545538531622

H 3.37538531112772

H -1.75729143577613

H -0.56699972284121

H 1.86603373298538
3.58176631576783

10.85772187428851

9.82544080844156

10.58799777105038

8.96018659270884

5.17198509246087

6.51887177485013

7.27258828143550

8.60650546096027

5.99498884893887

2.16284812515165

4.06569286900776

4.32886391497318

3.46543753284757

1.65028357498425

3.81424266586637

2.71646274823163

0.85209212813935

1.05973160449392

0.57007190681881

0.98017465114380

4.08568572086753

2.94996098178689

1.57384436902427

2.04508497237558

3.49609444952047

4.18688876971118

4.33033909853616

5.45039321528447

6.74053320842058

6.95943965290644

1.37486526284960

2.27589097114016

4.75167878254729

4.17964681093336

4.14064191529483 2.50058755383820

3.24561485715988

1.72625671598459

0.29023003698833

1.83127870667921

0.11150379071806

3.62736087800479

1.44249431666935

2.72245573671568

4.03400959718271

4.82757310354905

3.28767664380482

1.57023463944480

4.96622993946454

10.64675383876911

11.11755066867651

10.25808037575066

11.41328321112714

9.26577083487684

6.72013816359160

5.74653289927648

8.11135806013111

1.47472974755166

1.62516692975344

2.64435483158835

3.38265484112804

2.40719395339668

0.89642001995050

1.07622761238769
12.90674238369809

12.14171322313475

9.86702615546761

11.46117469052590

11.23070178919638

9.99487864341586

8.05492750852378

13.38037219839283

11.84423900808437

14.79696628727504

15.24923078362580

15.40068169670575

13.65650446025784

13.28869029294389

11.72130098509878

8.82346893303607

8.19129186395790

11.55028848117236

9.73122008939108

9.25943298632948

7.56585196005179

9.53172807208513

7.72521434750997

14.92369394929217

15.20361728417009

13.55981504413652

12.01552650347691

10.46711655347880

8.65017047811102

7.00659498635537

13.67500273864719

14.66512090812275

16.60438840497223

14.92852110170134

16.75406426330938

13.96562196327928

12.59407860081630

7.25013748520012

8.51603810240829

9.99960739918877

7.01098052927596

7.01620783323634

7.30311920409318

16.09912782810133

14.70880353768188

13.16458256876638

8.06246760600904

17.36232928281751

17.06997254182704

17.33546223035272

12.98581669855227

12.26767221054241

13.79669269265173

13.37416137214590

11.13329248467548

14.12716420314506

11.79005689206178

8.33296989393793

7.82754207117508

6.02859244521696

15.21239408959772

18.41583172704388

12.73819147739074

11.17068804512553

10.88592143049250 


\section{References}

(1) CrysAlisPro Software System, Agilent Technologies UK Ltd, Yarnton, Oxford, UK, 2013.

(2) O. V. Dolomanov, L. J. Bourhis, R. J. Gildea, J. A. K. Howard, H. Puschmann, J. Appl. Cryst., $2009,42,339$.

(3) G. M. Sheldrick, Acta Cryst., 2008, A64, 112.

(4) A. Spek, J. Appl. Cryst. 2003, 36, 7.

(5) F. Neese, Wiley Interdiscip. Rev. Comput. Mol. Sci., 2012, 2, 73.

(6) F. Weigend and R. Ahlrichs, Phys. Chem. Chem. Phys., 2005, 7, 3297.

(7) A. Schäfer, H. Horn and R. Ahlrichs, J. Chem. Phys., 1992, 97, 2571.

(8) (a) S. Grimme, S. Ehrlich, L. Goerigk, J. Comput. Chem., 2011, 32, 1456. (b) S. Grimme, J. Antony, S. Ehrlich, H. Krieg, J. Chem. Phys., 2010, 132, 154104.

(9) (a) Neese, et al. ORCA manual, version 3.0; Max-Planck-Institute for Chemical Energy Conversion: Mülheim a. d. Ruhr, Germany, 2012; p 509. (b) P. O. Löwdin, J. Chem. Phys., 1950, 18, 365. (c) P. O. Löwdin, Adv. Quantum Chem., 1970, 5, 185.

(10) A.-R. Allouche, J. Comput. Chem., 2011, 32, 174. 